\title{
Brote de bacteriemia por E. faecalis en un servicio de inmunología pediátrica en un hospital de tercer nivel
}

\author{
Dra. Esther Lombardo-Aburto, ${ }^{*}$ Dr. Napoleón González-Saldaña, ${ }^{* *}$ Dr. José Luis Castañeda-Narváez, ${ }^{* * *}$ \\ Dr. Víctor Manuel Hernández-Bautista, ${ }^{* * * *}$ Dra. Hilda G. Hernández-Orozco***
}

\section{RESUMEN}

La identificación temprana de brotes de bacteriemia es una de las ventajas de la vigilancia epidemiológica de rutina. El Enterococcus es una bacteria de baja patogenicidad que forma parte de la flora normal de la vía gastrointestinal y genitourinaria. El objetivo de este estudio fue describir un brote intrahospitalario de tres casos de bacteriemia por E. faecalis en el Servicio de Inmunología detectados entre el 6 y 9 de agosto del 2009. Todos respondieron al tratamiento con ampicilina. Se analizaron como factores de riesgo el uso de métodos y procedimientos invasivos, medicamentos prescritos, ubicación del paciente en su servicio, personal que atendió a los pacientes. El control del brote se logró mediante reforzamiento de precauciones estándar y específicas de contacto, de capacitación de medidas de prevención en el personal y de la vigilancia epidemiológica del área. Se sugiere reevaluar la localización de los pacientes e incrementar las estrategias de prevención de infecciones nosocomiales en el personal.

Palabras clave: Brote, E. faecalis, brote de bacteriemia, infecciones nosocomiales, inmunocomprometidos.

\section{ABSTRACT}

Early identification of bacteremia outbreaks is one of the advantages of routine epidemiological surveillance. Enterococcus is a low pathogenic bacteria and part of the normal flora of the gastrointestinal and genitourinary tracts. The aim of this study was to report a nosocomial outbreak of 3 cases of bacteremia due to $E$. faecalis detected in the Department of Immunology from August 6 to August 9, 2009. All cases responded to ampicillin. We analyzed the following potential risk factors: invasive methods and procedures, prescribed drugs, patient location, personnel in charge of the patients. Control of the outbreak was achieved through strengthening of standard precautions and specific contact, strengthening of prevention training of the staff and epidemiological surveillance of the area. We emphasize the importance of evaluating the location of patients and the enhancement of prevention strategies of nosocomial infections.

Key words: Outbreak, E. faecalis, outbreak of bloodstream infection, immunocompromised patients, nosocomial infections.

* Médico Adscrito Servicio Epidemiología

** Jefe de Servicio de Infectología. Comité de Infecciones Nosocomiales

*** Médico Adscrito Infectología. Comité de Infecciones Nosocomiales

**** Jefe de Servicio de Inmunología

***** Grupo de Control de Infecciones Nosocomiales (Dra. Leticia Azcarrunz, QFB Patricia Arzate Barbosa, Enf Elvira Solórzano García, Enf Aurora Rosas Ruiz, Enf Esperanza Lucas Resendiz)

Instituto Nacional de Pediatría

Correspondencia Dra. Hilda G Hernández-Orozco. Instituto Nacional de Pediatría. Insurgentes Sur 3700C Col. Insurgentes Cuicuilco. México 04530 D.F. zhongu10@yahoo.com.mx

Recibido: enero, 2010. Aceptado: febrero, 2010.

Este artículo debe citarse como: Lombardo-Aburto E, GonzálezSaldaña N, Castañeda-Narváez JL y col. Brote de bacteriemia por $E$. faecalis en un servicio de inmunología pediátrica en un hospital de tercer nivel. Acta Pediatr Mex 2010;31(2):67-69.

www.nietoeditores.com.mx

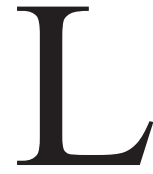

os enterococos son cocos gram positivos, catalasa negativos y anaerobios facultativos; las especies productoras de infección son los Enterococcus faecalis y en una proporción mucho menor de 9:1, E. faecium; los otros, E. casseliflavius, E. gallinarium, E. durans, E. hirae, E. raffinosus y E. avium rara vez se aíslan de muestras clínicas. La colonización por estas bacterias es frecuente en pacientes en unidades de cuidados intensivos y es un factor predisponente de septicemia. ${ }^{1-2}$

El enterococo es una causa de infección intrahospitalaria; su hábitat es el tubo digestivo; existe en las heces de adultos en $>90 \%$ de los casos. Los neonatos se colonizan en $50 \%$ de los casos a la semana de edad. La transmisión directa del germen, de persona a persona es un mecanismo importante de transmisión en brotes de septicemia en 
neonatos y adultos en unidades de cuidados intensivos ${ }^{3-4}$ La identificación reciente de brotes causados por E. faecalis hace necesario considerar como responsable a este microorganismo de baja patogenicidad, ya que es parte de la flora microbiana normal del tuvo digestivo y de la vía urinaria como un patógeno emergente de infección intrahospitalaria. El 6\% de las bacteriemias con o sin endocarditis se deben a este grupo de bacterias; su transmisión nosocomial es a través de las manos o guantes a partir de pacientes infectados o colonizados. ${ }^{-5-6}$

Se han hallado altos niveles de resistencia de los $E$. faecalis a los aminoglucocidos y la estreptomicina. ${ }^{3} \mathrm{Los}$ enterococos tienen resistencia intrínseca a las cefalosporinas, la clindamicina y el cotrimoxazol. La resistencia adquirida a los betalactámicos se debe a la modificación de las proteínas fijadoras de penicilina (PBP-5) y a la producción de betalactamasas; el primer mecanismo es excepcional en E. faecalis.

La resistencia intrínseca a los aminoglucósidos es de bajo grado, mientras que la adquirida se debe a la producción de enzimas modificadoras de los aminoglucócidos. Sin embargo, cada vez es más frecuente la aparición de cepas resistentes a los glucopéptidos (vancomicina). Los enterococos resistentes a vancomicina han sido causa de transferencia horizontal de cepas de Staphylococcus aureus con menor sensibilidad a la vancomicina. ${ }^{2}$

Generalmente se recomienda seguir las precauciones estándar en cualquier paciente con infección sistémica por E. faecalis y en caso de niños colonizados con enterococos resistentes a la vancomicina conviene seguir además precauciones específicas de aislamiento de contacto. ${ }^{7} \mathrm{El}$ objetivo de este trabajo es informar un brote de bacteriemia por E. faecalis en el Servicio de Inmunología del Instituto Nacional de Pediatría (INP).

\section{ESTUDIO DE BROTE}

Se estudió un brote de bacteriemia en el Servicio de Inmunología del INP, del 6 al 9 de agosto del 2009. Los casos probables fueron pacientes que tuvieron fiebre. Los casos confirmados fueron los pacientes con diagnóstico clínico de bacteriemia causada por E. faecalis corroborado por hemocultivo. Se analizaron las posibles fuentes de transmisión del brote. Se determinó la tasa de ataque de bacteriemia por E. faecalis en el Servicio de Inmunología.
La investigación encontró tres casos de bacteriemia por E. faecalis. Se confirmó que se trataba de un brote por el incremento en la frecuencia de bacteriemia en el Servicio, en el que no habían ocurrido bacteriemias por E. faecalis en los últimos dos años.

Generalmente se debe realizar seguimiento al menos por dos períodos de incubación de la enfermedad para dar por terminado el brote; en este caso se decidió un mes de seguimiento.

Dos de los casos de bacteriemia fueron del sexo masculino. La tasa de ataque en el Servicio de Inmunología es de $42.8 \%$ : dos casos en menores de cuatro años y uno en un paciente de 16 años. Todos los pacientes evolucionaron satisfactoriamente y sin complicaciones. En los tres casos se aisló $E$. faecalis sensible a la ampicilina y vancomicina. El hecho de que en los tres casos el microorganismo tuviera la misma sensibilidad, orienta a pensar en la existencia de un brote en el servicio aunado al incremento de la frecuencia de estos casos en el servicio.

Las medidas de control fueron precauciones específicas de aislamiento de contacto en los casos y la obtención de hemocultivos negativos; reforzamiento de precauciones estándar -higiene de manos- y capacitación del personal del servicio para reforzar las medidas de prevención de infecciones nosocomiales.

Se supervisó estrechamente la aplicación de procedimientos de limpieza en las áreas. No se logró identificar la fuente del brote; se piensa que probablemente fue la transmisión a través de las manos de personas que tuvieron interacción con los pacientes.

Los E. faecalis aislados eran sensibles a la ampicilina y la vancomicina. Debido al tipo de paciente inmunocomprometido, se decidió continuar el tratamiento empírico iniciado: dos casos fueron tratados con vancomicina y un caso con teicoplamina. Se continuó la vigilancia hasta el 9 de septiembre. No hubo nuevos casos.

\section{DISCUSIÓN}

La mayoría de las infecciones por E. faecalis ocurren en individuos con ruptura en las barreras físicas normales, con hospitalización prolongada; por el uso previo de antibióticos y por compromiso del sistema inmunológico ${ }^{8}$. Nuestros pacientes tenían estas tres características particulares y el promedio de hospitalización era de más a 150 días; habían recibido tratamiento con antibióticos antes 
de la presentación de la bacteriemia. Todos tuvieron un catéter venoso central en la vena subclavia o en la yugular; ninguno tuvo un catéter en la vena femoral que pudiera relacionarse con episodio diarreico.

Aunque los brotes por enterococos informados en pacientes inmunocomprometidos son por microorganismos resistentes ${ }^{9}$, en este caso eran sensibles a la ampicilina. Esto es similar a lo descrito en un estudio de sensibilidad realizado en un hospital de oncología donde se encontró que el E. faecalis y el E. faecium fueron sensibles a la ampicilina en 73 y $16 \%$ respectivamente y que para la vancomicina fueron sensibles el 100\% en ambas especies. Estos resultados representaron el $2.9 \%$ de los aislamientos ${ }^{8}$.

$\mathrm{El}$ incremento en los patrones de resistencia antimicrobiana, como resultado del uso creciente de antibióticos, es causa de fallas en los esquemas antimicrobianos, debido a lo cual en ocasiones se inicia un tratamiento empírico con antibióticos de amplio espectro. Esto debe ser valorado en el área que se presente, de acuerdo al patrón de sensibilidad antimicrobiana de E. faecalis como se halló en este estudio del brote. Conocer el patrón de sensibilidad de los microorganismos permite el empleo de tratamientos empíricos tempranos para evitar que ocurra resistencia antimicrobiana por el uso innecesario inicial de antibióticos de amplio espectro.

\section{CONCLUSIÓN}

Lo más importante de una vigilancia epidemiológica para prevenir infecciones nosocomiales es la detección temprana de los brotes; la identificación y el seguimiento de casos aislados de infección intrahospitalaria y la identificación de los factores de riesgo. Esto permite como en este caso, que se detecte rápidamente un brote y se controle de inmediato. También permite prevenir casos y brotes futuros. Para ello es vital la participación activa de todos los servicios involucrados, especialmente el grupo medico y de enfermería del servicio ya que ellos son los que determinan muchas veces que ocurran casos inusuales, lo que permite realizar la detección de casos por el Comité de Infecciones y la implementación de medidas de control y prevención, que sería imposible llevar a cabo sin la participación de todo trabajador del Instituto.

Desafortunadamente, no se realizaron estudios de biología molecular a los enterococos aislados, por lo que no se pudo tener evidencia concluyente que asegurara la presencia de un brote.

\section{REFERENCIAS}

1. Salas VAV, Boza CR, Bustamante GR, et al. Prevalencia e identificación genotípica de Enterococos vancomicina resistente en pacientes en un medio hospitalario. Acta Med Costarric. 2004; 46(1):19-26.

2. Cause M, Franco ADA, García FCM, Rodríguez FC, Casal M. Sensibilidad a los antimicrobianos de Enterococcus faecalis aislados de pacientes en la provincia de Córdoba. Rev Esp Quimioterap. 2006; 19(2):140-3.

3. Haslam DB, St Geme III JW Enterococcus species. In: Long SS, Pickering LK, Prober G CH. Pediatric infectious disease. 3ed. Elsevier; 2008.p.716-9.

4. Arellano GJ, Garzón JT, Giono SC, Mateos OS. High level aminoglycoside resistance enterococcus spp in a tertiary care hospital in Mexico. Electronic J Biomedicine. 2005;1:40-5.

5. Ponessa A, Gambandé T, All L, Fernández GA et al. Enterococcus vancomicine resistentes: colonización en pacientes hospitalizados, en Rosario Argentina. Acta Bioquím Clin Latinoam. 2006;40(4):587-90.

6. American Academy of Pediatrics. Estreptocócica y enterocócicas infecciones. En: Pickering LK. Ed. 2003 Red book Report of the Committee on Infectious Diseases. 26ed. Elk Grove Village IL: American Academy of Pediatrics; 2003.p. 324-6.

7. Varejão TM, Cais DP, Zeigler R, cols. Clustering of Enterococcus faecalis infections in a Cardiology Hospital Neonatal Intensive Care Unit. Braz J Infect Dis. 2006;10(2):113-6.

8. Cornejo PJ, Velazquez CA, Diaz AG, Volkow PF. Tendencia del perfil de sensibilidad antimicrobiano de los aislamientos de sangre en un hospital oncológico (1998-2003). Salud Pub Méx. 2005;47:288-93.

9. Drews SJ, Richardson SE, Wray R, et al. An outbreak of vancomycin-resistant Enterococcus faecium in an acute care pediatric hospital: Lessons from environmental screening and a case-control study. Can J Infect Dis Med Microbiol 2008;19(3):233-336.

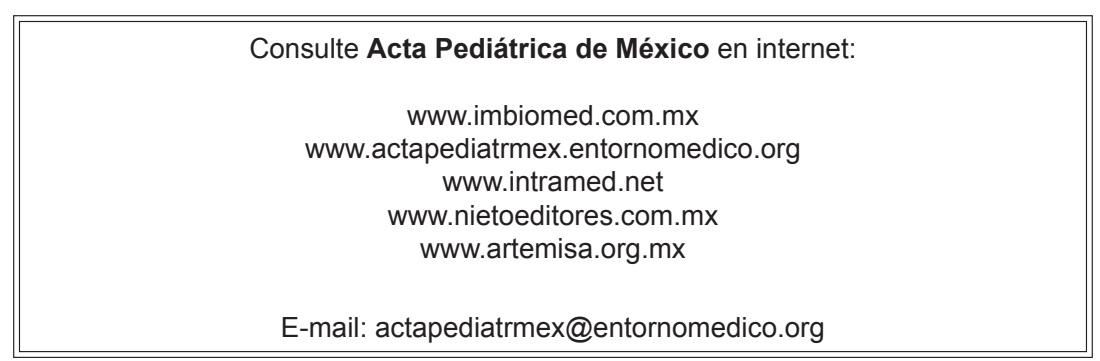

